\title{
Immune regulatory markers of lepidic-pattern adenocarcinomas presenting as ground glass opacities
}

\author{
David B. Nelson ${ }^{1}$, Kyle G. Mitchell ${ }^{1}$, Jing Wang ${ }^{2}$, Junya Fujimoto ${ }^{3}$, Myrna Godoy ${ }^{4}$, Carmen Behrens ${ }^{5}$, \\ Xiaofeng Zheng ${ }^{2}$, Jianjun Zhang ${ }^{5}$, Boris Sepesi ${ }^{1}$, Ara A. Vaporciyan ${ }^{1}$, Wayne L. Hofstetter ${ }^{1}$, \\ Reza J. Mehran ${ }^{1}$, David C. Rice ${ }^{1}$, Garrett L. Walsh ${ }^{1}$, Stephen G. Swisher ${ }^{1}$, Cesar A. Moran ${ }^{6}$, \\ Neda Kalhor ${ }^{6}$, Annikka Weissferdt ${ }^{6}$, Ignacio I. Wistuba ${ }^{4}$, Jack A. Roth ${ }^{1}$, Mara B. Antonoff ${ }^{1}$
}

${ }^{1}$ Department of Thoracic and Cardiovascular Surgery, ${ }^{2}$ Deparment of Bioinformatics and Computational Biology, ${ }^{3}$ Department of Translational Molecular Pathology, ${ }^{4}$ Department of Diagnostic Radiology, ${ }^{5}$ Department of Thoracic/Head and Neck Medical Oncology, ${ }^{6}$ Department of Pathology, University of Texas MD Anderson Cancer Center, Houston, TX, USA

Contributions: (I) Conception and design: DB Nelson, KG Mitchell, J Wang, B Sepesi, AA Vaporciyan, WL Hofstetter, RJ Mehran, DC Rice, II Wistuba, JA Roth, MB Antonoff; (II) Administrative support: B Sepesi, AA Vaporciyan, WL Hofstetter, RJ Mehran, DC Rice, GL Walsh, SG Swisher, JA Roth, MB Antonoff; (III) Provision of study materials or patients: J Fujimoto, M Godoy, C Behrens, X Zheng, J Zhang, B Sepesi, AA Vaporciyan, WL Hofstetter, RJ Mehran, DC Rice, GL Walsh, SG Swisher, CA Moran, N Kalhor, A Weissferdt, II Wistuba, JA Roth, MB Antonoff; (IV) Collection and assembly of data: DB Nelson, KG Mitchell, J Wang, J Fujimoto, M Godoy, C Behrens, X Zheng; (V) Data analysis and interpretation: DB Nelson, KG Mitchell, J Wang, J Fujimoto, M Godoy, C Behrens, X Zheng, J Zhang, B Sepesi, II Wistuba, JA Roth, MB Antonoff; (VI) Manuscript writing: All authors; (VII) Final approval of manuscript: All authors.

Correspondence to: Mara B. Antonoff, MD. Department of Thoracic and Cardiovascular Surgery, University of Texas MD Anderson Cancer Center, 1515 Holcombe Blvd, Houston, TX 77030, USA. Email: mbantonoff@mdanderson.org.

Background: The tumor immune microenvironment of lepidic-pattern adenocarcinoma remains poorly understood. In this study, we characterized tumor infiltrating lymphocytes (TILs) and percent PD-L1 expression among adenocarcinoma presenting as either radiographic ground glass opacities (GGOs) or solid lesions.

Methods: Pathologic specimens of patients with clinical stage I lung adenocarcinoma were analyzed using tissue microarray sectioning. The invasive portion of the tumor was selected for the tissue core. Lepidic growth pattern was confirmed among the GGO lesions using whole section analysis. Progression was defined as $\mathrm{pN}+$ or subsequent recurrence.

Results: A total of 181 patients were identified, among whom 13 (7\%) represented GGOs without clinical progression, 113 (62\%) represented radiographic solid lesions that never progressed, and 55 (30\%) represented radiographic solid lesions that ultimately did progress. CD57+ cell density, a marker for antigenspecific, oligoclonal T cells and NK cells, differed among the three cohorts, with the highest cell density observed within radiographically solid lesions without progression, and lower cell density both in the radiographic solid lesions that progressed and GGOs. Other TIL phenotypes were not statistically different between cohorts. Of substantial clinical interest, median percent PD-L1 positive cells within GGOs was 14, whereas that of radiographic solid lesions without progression was 22, and radiographic solid lesions that subsequently progressed was $27(\mathrm{P}=0.07)$.

Conclusions: Lepidic pattern adenocarcinoma presenting as GGOs and radiographic solid lesions show differential immune regulation. Further studies to investigate whether GGOs representing adenocarcinoma have varying susceptibility to immune checkpoint inhibitor therapy are warranted.

Keywords: Non-small cell lung cancer (NSCLC); ground-glass opacity (GGO); immunotherapy

Submitted Aug 25, 2019. Accepted for publication Dec 26, 2019.

doi: $10.21037 /$ jtd.2020.01.42

View this article at: http://dx.doi.org/10.21037/jtd.2020.01.42 


\section{Introduction}

The immune regulatory environment has been shown to have vast importance with regard to determining prognosis of early stage non-small cell lung cancer (NSCLC) (1) as well as for the treatment of metastatic NSCLC $(2,3)$. Programmed death ligand 1 (PD-L1) binds to programmed death protein 1 (PD-1) on cytotoxic T-cells to allow for tumor cell escape from cytotoxic T-cell activity. Although PD-L1 is the most well studied immune biomarker, other components of the tumor immune microenvironment including $\mathrm{CD} 3+, \mathrm{CD} 4+, \mathrm{CD} 8+$, and FOXP3+ T-cell infiltration have been shown to have important prognostic value $(4,5)$. These cells, as well as other important components of the innate and adaptive immunity, are active in the native lung and provide an important defense against frequent antigenic exposure during respiration $(6,7)$. The tumor immune microenvironment can either lead to tumor suppressing activity, or, once maladapted by the tumor, may ultimately lead to tumor progression. The microenvironment of lepidic pattern adenocarcinoma is also unique, with less stromal cells known to promote cancer cell invasion and metastasis $(8,9)$. Long-term survival for lepidic predominant adenocarcinoma appears more favorable than other subtypes and is $86 \%$ at five-years (10). Although the tumor immune microenvironment has been a well-studied regulator towards either preventing or promoting tumor progression, its characterization specifically in the setting of lepidic predominant adenocarcinoma remains poorly understood. We hypothesized that immune cell infiltration would show a continuum of results from adenocarcinoma presenting as ground glass opacities (GGOs), to those presenting as radiographically solid tumors without progression, and to those presenting as radiographically solid tumors with progression. We defined progression as $\mathrm{pN}+$ or subsequent recurrence during follow-up. Between cohorts, we specifically compared tumor infiltrating lymphocytes (TILs) and percent PD-L1 positive cells. We additionally performed additional whole section histologic review among the GGO specimens to confirm lepidic growth pattern.

\section{Methods}

\section{Patient selection}

This study was approved by the MD Anderson Cancer Center Institutional Review Board with a waiver of individual informed consent (PA15-0167). A prospectively maintained departmental database was queried to select patients with clinical stage I lung adenocarcinoma who underwent resection with curative intent and who had tissue microarray data available for analysis. Tissue microarray data were previously performed on specimens from patients between the years 2006-2009. The seventh edition of the American Joint Commission on Cancer staging system was used for staging purposes.

\section{Variable definitions and radiographic and pathologic review}

Progression was defined as $\mathrm{pN}+$ or subsequent recurrence during follow-up. Among patients who presented with pure or mixed GGOs on preoperative imaging, additional whole section histologic review was performed to confirm the presence of lepidic growth pattern.

\section{Immunobistochemical staining and image analysis}

Tissue microarrays was performed using formalin-fixed, paraffin-embedded tumor blocks as has been previously described (11,12). In brief, tissue microarray sections were prepared using three $1.0-\mathrm{mm}$ tissue cores obtained from the center, the mid-periphery, and periphery of the tumor and selected to represent invasive adenocarcinoma. Fourmicron thick sequential histologic tumor sections were selected from representative tumor blocks. An automated staining system was used to perform analysis (BONDMAX, Leica Microsystems, Vista, CA, USA). Antibodies used were as follows: CD3 (T-cell lymphocytes; dilution 1:100; Dako, Carpinteria, CA), CD4 (helper T cell; Novocastra, Leica Microsystems, Milton Keynes, UK; clone 4B12, dilution 1:80; Leica Biosystems, Buffalo Grove, IL, USA), CD8 (cytotoxic T cell; clone CD8/144B, dilution 1:20; ThermoFisher Scientific, Inc., Waltham, MA, USA), CD57 (natural killer T cell; clone HNK-1, dilution 1:40; BD Biosciences, San Jose, CA, USA), CD45RO (memory T cell; clone UCHL1, ready to use; Leica Biosystems), FOXP3 (regulatory T cell; clone 206D, dilution 1:50; BioLegend, San Diego, CA, USA), PD-L1 (clone E1L3N, dilution 1:100; Cell Signaling Technology, Beverly, MA, USA), and PD-1 (clone EPR4877-2, dilution 1:250; Abcam, Cambridge, MA, USA). Marker expression was detected using a Novocastra Bond Polymer Refine Detection kit (Leica Microsystems) with a diaminobenzidine reaction for detection of antibody labeling and hematoxylin counterstaining. The positive and negative controls used 


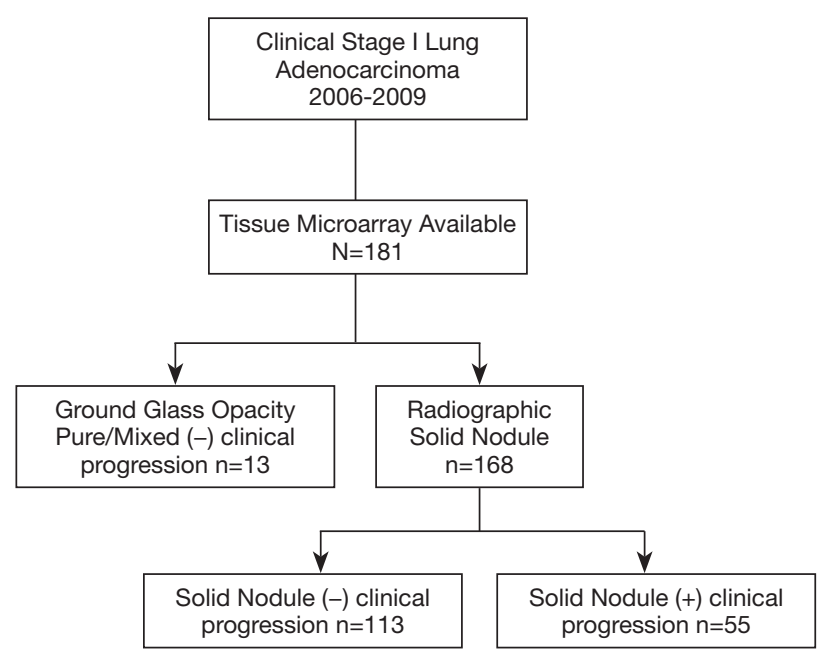

Figure 1 Study flowchart indicating patient selection criteria.

for PD-L1 IHC expression were human embryonic kidney 293 cell line transfected or not transfected with PD-L1 gene. The positive and negative controls used for TIL IHC expression were human tonsil FFPE tissues with and without primary antibody. Controls were performed with each iteration of IHC staining.

Imaging analysis was performed using slides from immunohistochemically stained TMA sections that were digitally scanned at $\times 200$ magnification using a ScanScope Aperio AT Turbo slide scanner (Leica Microsystems). Visualization of the images was performed using the ImageScope software program (Leica Microsystems) with automated cell counting and the Aperio Image Toolbox (Leica Microsystems) was used for analysis. All image analysis was performed with the pathologist being blinded to patients' outcome. The densities of cells expressing CD3, CD4, CD8, CD57, CD45RO, PD-L1, and FOXP3 were evaluated using the Aperio nuclear algorithm, with the cells positive in 5 random square areas $\left(1 \mathrm{~mm}^{2}\right.$ each) being counted in both intratumoral and peritumoral compartments. Counting was automated and was not stratified by intratumoral or peritumoral compartment, or selected location of the tumor. Histologic assessment of each $1 \mathrm{~mm}^{2}$ was performed to verify that the tumor tissue was included in the selected intratumoral region, which was assured to represent at least $80 \%$ malignant cells and tumor stroma. The peritumoral compartment consisted of only non-malignant cells. Each area examined was overlapped with the sequential IHC slides to quantify each marker at the same location of the tumor specimens. The average total number of cells positive for each marker in the 5 square areas was expressed in density per $\mathrm{mm}^{2}$.

\section{Statistical analysis}

Differences in patient or tumor characteristics were compared with one-way analysis of variance (ANOVA), chisquared, or Fischer's exact test, where appropriate. Among outcome variables, Wilcoxon rank sum test was used to compare cohorts with log-transformation. Heatmaps were generated using unsupervised clustering in order to identify patterns of immune cell infiltrates according to each cohort. One-sided Cuzick's test was used to test for an ordinal relationship between percent positive PD-L1 cells and each cohort. $\mathrm{P}$ values were considered statistically significant with $\mathrm{P}<0.05$. All statistical tests were two-sided unless otherwise specified.

\section{Results}

\section{Patient and tumor characteristics}

181 patients were identified as having stage I lung adenocarcinoma and had immune microenvironment tissue microarray data available (Figure 1). Thirteen (7\%) represented pure or mixed GGOs that never progressed, 113 $(62 \%)$ represented radiographically solid lesions that never progressed, and 55 (30\%) represented radiographically solid lesions that ultimately progressed. Progression was defined as $\mathrm{pN}+$ or subsequent recurrence. Detailed patient, tumor and operative characteristics are provided in Tables 1 and 2. Median follow-up was 8.5 years among those with GGOs without progression, 8.5 years among those with radiographically solid lesions without progression, and 5.5 years among those with radiographically solid lesions with progression.

\section{Tumor immune microenvironment among lepidic-pattern adenocarcinoma presenting as GGOs}

The cell densities of several immune regulatory markers were compared within each cohort. Representative images of immunohistochemistry staining are shown in Figure 2. Unsupervised hierarchal clustering analysis is shown in Figure 3. There was no difference in the density of CD45Ro, a marker for memory T-cells (13), nor was there a difference in FOXP3, a marker for regulatory T-cells which facilitates immune evasion (14). Additionally, CD3+ 
Table 1 Patient, tumor and operative characteristics

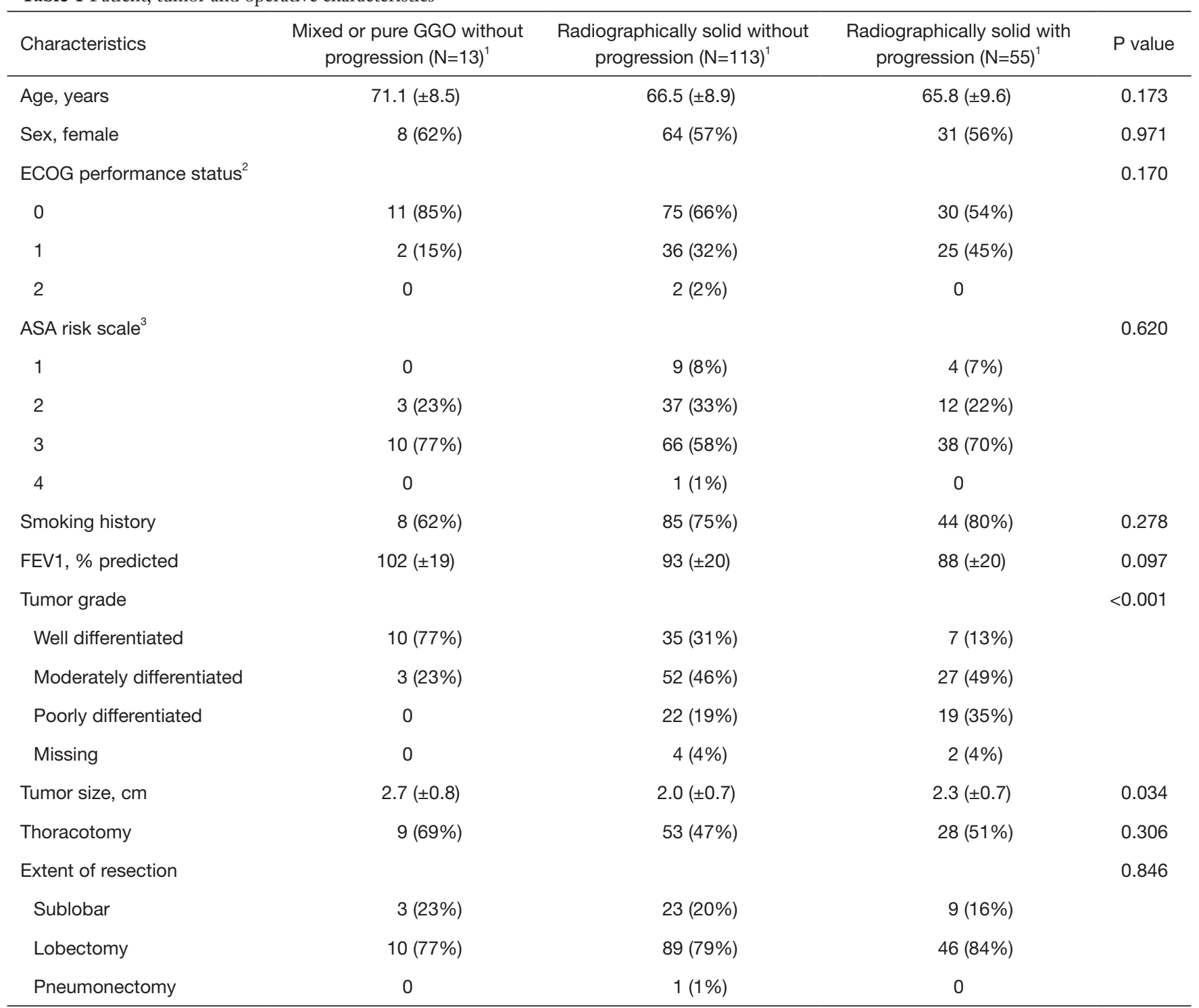

${ }^{1}$, data represent $\mathrm{N}$ (percentage) or mean ( \pm standard deviation); ${ }^{2}, \mathrm{~N}=12$ missing; ${ }^{3}, \mathrm{~N}=1$ missing. ECOG, Eastern Cooperative Oncology Group; ASA, American Society of Anesthesiologists; FEV1, forced expiratory volume in one second; GGO, ground glass opacity.

cell density and CD4+ cell density (markers for total T-cells and T-helper cells) were not different. However, CD57+ cell density, a marker for antigen-specific oligoclonal $\mathrm{T}$ cells and NK cells, differed among the three cohorts, with the highest cell density observed within radiographically solid lesions without progression, and lower cell density both in the radiographically solid lesions that progressed and
GGOs without progression (Figure 4).

Inbibitory checkpoint expression among lepidic-pattern adenocarcinoma

There was no difference in the distribution of percent PD-L1 positive cells within the tumor within each cohort $(\mathrm{P}=0.26$, Figure 4). However, when modeled as an ordinal 
Table 2 Detailed imaging characteristics of lepidic pattern adenocarcinoma presenting as ground glass opacities

\begin{tabular}{lc}
\hline Variable & $\mathrm{N}(\%)$ \\
\hline Total lesion size $(\mathrm{cm})$, mean $( \pm \mathrm{SD})$ & $2.7( \pm 0.8)$ \\
Size of solid component $(\mathrm{cm})$, mean $( \pm \mathrm{SD})$ & $1.3( \pm 1.1)$ \\
Nodule type & \\
Partial solid nodule & $10(77 \%)$ \\
Ground glass opacity & $3(23 \%)$ \\
Air-bronchogram & $9(69 \%)$ \\
Pseudo-cavitation & 0 \\
Cystic appearance & 0 \\
Spiculation & $3(23 \%)$ \\
Reticulation & $6(46 \%)$ \\
Lobulation & $8(62 \%)$ \\
SUVmax ${ }^{1}$, mean $( \pm \mathrm{SD})$ & $2.8( \pm 1.2)$ \\
\hline
\end{tabular}

${ }^{1}, 4$ patients with missing data. relationship, there was a non-significant trend toward increasing expression within each cohort, with the lowest expression being within GGOs without progression (median 14), followed by radiographically solid lesions without progression (median 22), and the highest among radiographically solid lesions that subsequently progressed (median 27, $\mathrm{P}=0.07$ ).

\section{Discussion}

We report that the tumor immune microenvironment of adenocarcinoma presenting as GGOs is unique, with decreased CD57+ cell density as well as a qualitative decline in percent PD-L1 positive cells as compared with radiographically solid lesions.

Despite the frequent identification of GGOs, the role of the tumor immune microenvironment within either pre-malignant lesions or conversely within lepidic pattern adenocarcinoma has been not well characterized to date. To
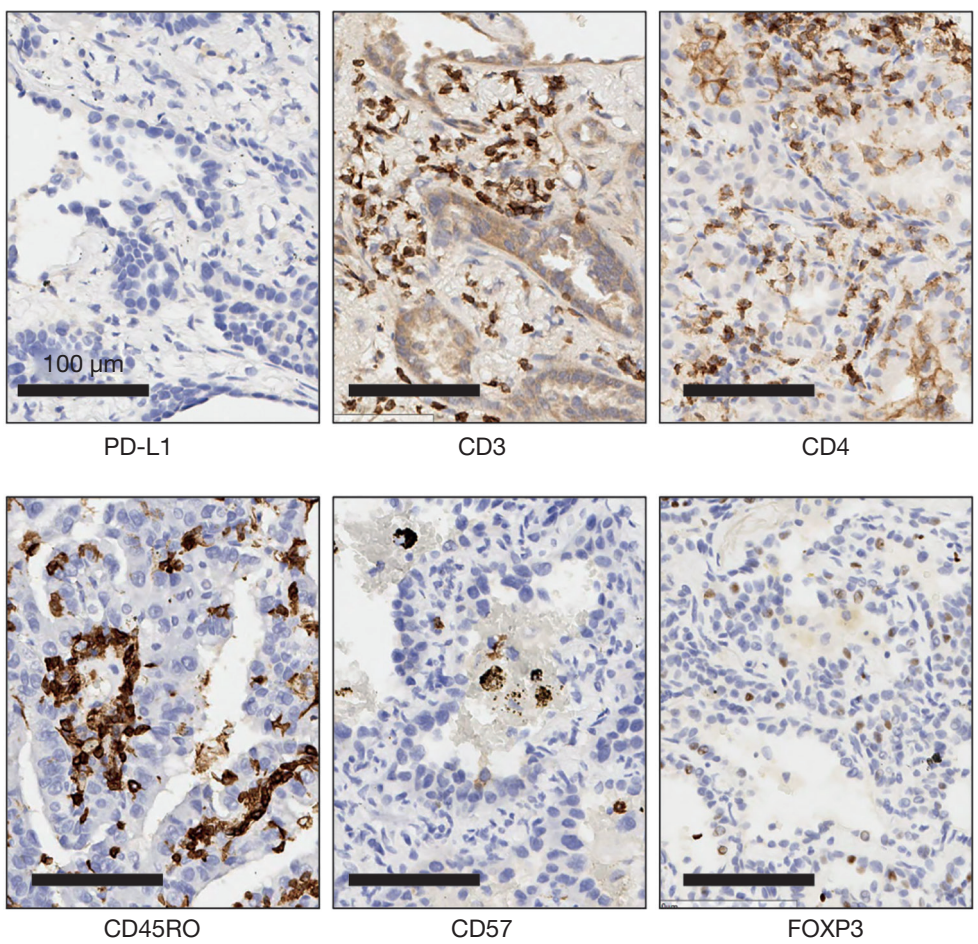

Figure 2 Representative scanned images of immunohistochemistry staining. Scale bar $=100 \mu \mathrm{m}$. 


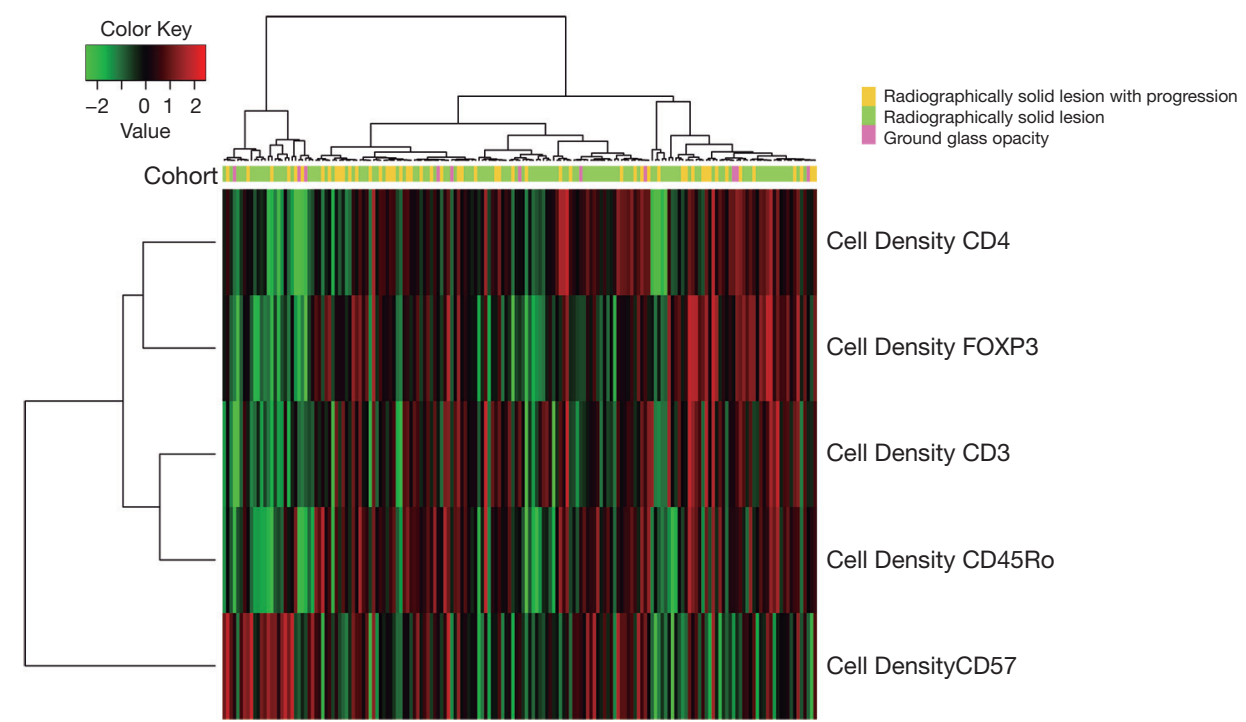

Figure 3 Associations between tumor microenvironment and (I) GGO without progression, (II) radiographically solid lesion without progression, and (III) radiographically solid lesion with progression. GGO, ground glass opacity.
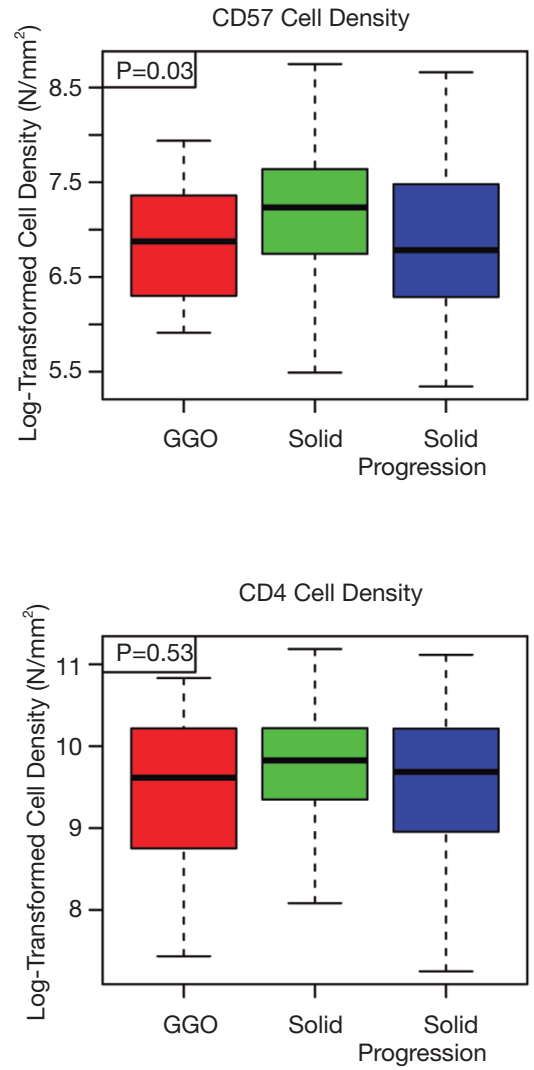
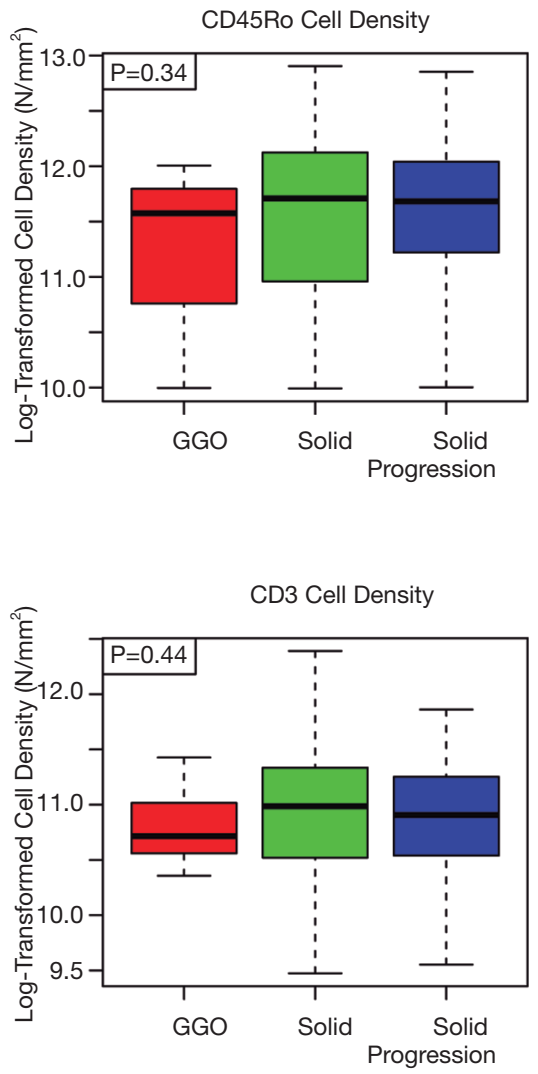

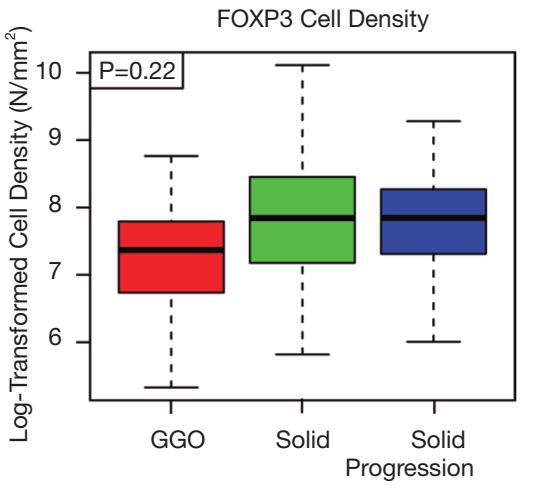

\% Positive Cells PD-L1 Tumor

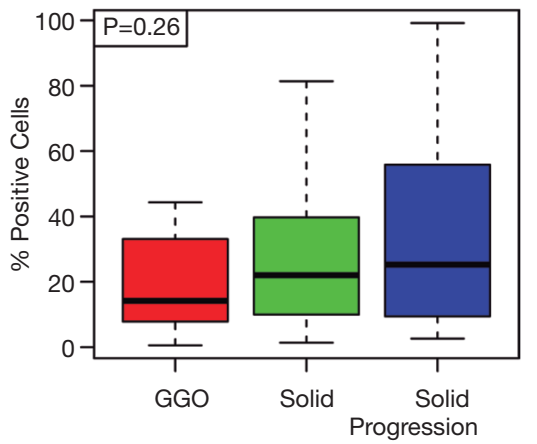

Figure 4 Boxplot of immune regulatory markers according to: (I) GGO without progression, (III) radiographically solid lesion without progression, and (III) radiographically solid lesion with progression. GGO, ground glass opacity. 
our knowledge, the only publication on PD-L1 expression within adenocarcinoma in situ (AIS) and minimally invasive adenocarcinoma (MIA) found that both AIS and MIA demonstrated poor PD-L1 expression (15). Further details, including the role of TILs, are lacking at this time. However, additional studies have highlighted differences in the tumor immune microenvironment of lepidic pattern adenocarcinoma, including a decrease in tumor-promoting podoplanin-positive cancer-associated fibroblasts and CD204-positive tumor-associated macrophages (9). In addition, a matched pair analysis revealed that the expression of hypoxia-related molecules were lower among lepidic pattern adenocarcinoma, including glucose transport and carbonic anhydrase IX (8).

Possibly related to changes in underlying biology as outlined above, there is also substantial evidence that GGOs are associated with improved prognosis. Improved survival has been observed among tumors that have a ground glass component (16) or those with a lepidic component $(17,18)$. Although progression is less common among tumors arising in GGOs, effort has been made toward identifying those patients with ground glass lesions or lepidic pattern adenocarcinoma who nonetheless progress. Factors that have been found to be associated with an increased risk of progression include prior history of lung adenocarcinoma, increasing size of the solid component of the ground glass opacity, presence of a micropapillary component, and lymphatic or vascular invasion (19-21).

A recently published meta-analysis including 26 studies revealed that intratumoral presence of CD57+ lymphocytes was significantly associated with improved overall survival among several solid tumor types, including NSCLC (22). CD57, also known as human natural killer-1 (HNK-1) and LEU-7, is present on CD8+ T-lymphocytes and natural killer (NK) cells and represents terminally differentiated cells with diminished proliferative capacity. CD8+ T-lymphocytes and NK cells that express CD57 demonstrate increased expression of cytolytic enzymes useful for cytolysis of tumor cells including granzyme A, granzyme $\mathrm{B}$, and perforin (23). In addition, the cytokine IFN-gamma, which inhibits tumor growth, has shown to be produced more readily by CD57+ cells (24). In our study, we found decreased CD57+ cell density within radiographically solid lesions that ultimately progressed, suggesting an escape from immune system activity within such stage I tumors. Interestingly, as compared with radiographically solid lesions that never progressed, we found that CD57+ activity was diminished within GGOs that never progressed, raising the question whether GGOs are immunologically inert.

There are several limitations of this retrospective analysis. Although our cohort includes 181 patients, only 13 of these represent GGOs, which limits the power for the study. Due to power constraints, we were unable to stratify based on several variables including tumor size, driver mutation status, or histologic subtype. In addition, we were unable to report marker co-localization for this study and staging was also limited to AJCC seventh edition. Lastly, with multiple comparisons, there is a risk of a type I error. However, our results are consistent with prior literature highlighting poor PD-L1 expression within AIS or MIA as discussed previously.

In conclusion, we found that adenocarcinoma presenting as GGOs demonstrate differential immune cell infiltration as compared with radiographically solid lesions. Further research to elucidate differences in immune regulation for lepidic pattern adenocarcinoma should be investigated. These findings will be especially helpful given the rise in use of immunotherapy and to determine if lepidic-pattern adenocarcinoma demonstrates varying susceptibility to immune checkpoint inhibitors.

\section{Acknowledgments}

Funding: This work was supported in part by the Cancer Prevention Research Institute of Texas Multi-Investigator Research Awards (RP160668, IIW), the National Institutes of Health/National Cancer Institute through the University of Texas Lung Specialized Programs of Research Excellence grant (P50CA70907, IIW), by generous philanthropic donations by the Mason family and anonymous donors, and by departmental funding.

\section{Footnote}

Conflicts of Interest: MBA serves as the unpaid editorial board member of Fournal of Thoracic Disease from Aug 2019 to Jul 2021. The other authors have no conflicts of interest to declare.

Ethical Statement: The authors are accountable for all aspects of the work in ensuring that questions related to the accuracy or integrity of any part of the work are appropriately investigated and resolved. This study was approved by the MD Anderson Cancer Center Institutional 
Review Board with a waiver of individual informed consent (PA15-0167).

Open Access Statement: This is an Open Access article distributed in accordance with the Creative Commons Attribution-NonCommercial-NoDerivs 4.0 International License (CC BY-NC-ND 4.0), which permits the noncommercial replication and distribution of the article with the strict proviso that no changes or edits are made and the original work is properly cited (including links to both the formal publication through the relevant DOI and the license). See: https://creativecommons.org/licenses/by-nc-nd/4.0/.

\section{References}

1. Suzuki K, Kadota K, Sima CS, et al. Clinical impact of immune microenvironment in stage I lung adenocarcinoma: Tumor interleukin-12 receptor beta2 (IL-12Rbeta2), Il-7R, and stromal FoxP3/CD3 ratio are independent predictors of recurrence. J Clin Oncol 2013;31:490-8.

2. Borghaei H, Paz-Ares L, Horn L, et al. Nivolumab versus docetaxel in advanced nonsquamous Non-Small-cell lung cancer. N Engl J Med 2015;373:1627-39.

3. Brahmer J, Reckamp KL, Baas P, et al. Nivolumab versus docetaxel in advanced squamous-cell non-small-cell lung cancer. N Engl J Med 2015;373:123-35.

4. Lavin Y, Kobayashi S, Leader A, et al. Innate immune landscape in early lung adenocarcinoma by paired singlecell analyses. Cell 2017;169:750-765.e17.

5. Geng Y, Shao Y, He W, et al. Prognostic role of tumorinfiltrating lymphocytes in lung cancer: A meta-analysis. Cell Physiol Biochem 2015;37:1560-71.

6. Altorki NK, Markowitz GJ, Gao D, et al. The lung microenvironment: an important regulator of tumour growth and metastasis. Nat Rev Cancer 2019;19:9-31.

7. Milette S, Fiset $\mathrm{P}, \mathrm{Walsh} \mathrm{L}$, et al. The innate immune architecture of lung tumors and its implication in disease progression. J Pathol 2019;247:589-605.

8. Katsumata S, Aokage K, Miyoshi T, et al. Differences of tumor microenvironment between stage I lepidic-positive and lepidic-negative lung adenocarcinomas. J Thorac Cardiovasc Surg 2018;156:1679-1688.e1672.

9. Naito M, Aokage K, Saruwatari K, et al. Microenvironmental changes in the progression from adenocarcinoma in situ to minimally invasive adenocarcinoma and invasive lepidic predominant adenocarcinoma of the lung. Lung Cancer
2016;100:53-62.

10. Russell PA, Wainer Z, Wright GM, et al. Does Lung Adenocarcinoma Subtype Predict Patient Survival? J Thorac Oncol 2011;6:1496-504.

11. Parra ER, Villalobos P, Zhang J, et al. Immunohistochemical and image analysis-based study shows that several immune checkpoints are co-expressed in non-small cell lung carcinoma tumors. J Thorac Oncol 2018;13:779-91.

12. Sepesi B, Cuentas EP, Canales JR, et al. Programmed death cell ligand 1 (pd-11) is associated with survival in stage I non-small cell lung cancer. Semin Thorac Cardiovasc Surg 2017;29:408-15.

13. Rauser S, Langer R, Tschernitz S, et al. High number of CD45RO+ tumor infiltrating lymphocytes is an independent prognostic factor in non-metastasized (stage I-IIa) esophageal adenocarcinoma. BMC Cancer 2010;10:608.

14. Hinz S, Pagerols-Raluy L, Oberg HH, et al. Foxp3 expression in pancreatic carcinoma cells as a novel mechanism of immune evasion in cancer. Cancer Res 2007;67:8344-50.

15. Yu R, He Z, Lou Y, et al. Clinical characteristics and programmed cell death ligand-1 expression in adenocarcinoma in situ and minimally invasive adenocarcinoma of lung. Oncotarget 2017;8:97801-10.

16. Hattori A, Matsunaga T, Takamochi K, et al. Prognostic impact of a ground glass opacity component in the clinical T classification of non-small cell lung cancer. J Thorac Cardiovasc Surg 2017;154:2102-2110.e1.

17. Strand TE, Rostad H, Strøm EH, et al. The percentage of lepidic growth is an independent prognostic factor in invasive adenocarcinoma of the lung. Diagn Pathol 2015;10:94.

18. Moon Y, Sung SW, Lee KY, et al. The importance of the lepidic component as a prognostic factor in stage I pulmonary adenocarcinoma. World J Surg Oncol 2016;14:37.

19. Kadota K, Villena-Vargas J, Yoshizawa A, et al. Prognostic significance of adenocarcinoma in situ, minimally invasive adenocarcinoma, and nonmucinous lepidic predominant invasive adenocarcinoma of the lung in patients with stage I disease. Am J Surg Pathol 2014;38:448-60.

20. Nelson DB, Godoy MCB, Benveniste MF, et al. Clinicoradiographic predictors of aggressive biology in lung cancer with ground glass components. Ann Thorac Surg 2018;106:235-41.

21. Shewale JB, Nelson DB, Rice DC, et al. Natural history 
of ground-glass lesions among patients with previous lung cancer. Ann Thorac Surg 2018;105:1671-7.

22. Hu G, Wang S. Prognostic role of tumor-infiltrating CD57-positive lymphocytes in solid tumors: A metaanalysis. Oncotarget 2017;9:8111-9.

23. Chattopadhyay PK, Betts MR, Price DA, et al. The cytolytic enzymes granyzme a, granzyme b, and

Cite this article as: Nelson DB, Mitchell KG, Wang J, Fujimoto J, Godoy M, Behrens C, Zheng X, Zhang J, Sepesi B, Vaporciyan AA, Hofstetter WL, Mehran RJ, Rice DC, Walsh GL, Swisher SG, Moran CA, Kalhor N, Weissferdt A, Wistuba II, Roth JA, Antonoff MB. Immune regulatory markers of lepidicpattern adenocarcinomas presenting as ground glass opacities. J Thorac Dis 2020;12(3):329-337. doi: 10.21037/jtd.2020.01.42 perforin: Expression patterns, cell distribution, and their relationship to cell maturity and bright CD57 expression. J Leukoc Biol 2009;85:88-97.

24. Lopez-Vergès S, Milush JM, Pandey S, et al. CD57 defines a functionally distinct population of mature NK cells in the human CD56dimCD16+ NK-cell subset. Blood 2010;116:3865-74. 